\title{
Analysis of Income Difference among Rural Residents in China
}

\author{
Yan Xue, Yeping Zhu, and Shijuan Li \\ Laboratory of Digital Agricultural Early-warning Technology of Ministry of \\ Agriculture of China, Institute of Agricultural Information, CAAS, \\ 100081 Beijing, China \\ \{Xueyan, Zhuyp\}mail.caas.net.cn
}

\begin{abstract}
This paper studies and analyzes the income difference among Chinese rural residents from 1997 to 2008 through absolute difference indices and relative difference indices. It comes to the conclusion that the absolute income difference among rural residents in China has been increasing year by year, while the relative difference remains around the average level and tends to increase in fluctuations in recent years. The paper also discusses the results and proposes corresponding countermeasures.
\end{abstract}

Keywords: Income Difference, Rural Residents, Analysis.

\section{Introduction}

Since the reform and opening up, China's economy has maintained a momentum of rapid development. But the income of residents, especially those in rural areas, has not increased along with the economy, causing a big gap between productivity and consumption level, which inevitably hampers the sustainable economic development. Therefore, to keep residents' income growth in line with economic development has both social and economic significance. As China is a large agricultural country, rural residents' income growth is especially important, which is also the starting point of this study.

\section{Measurement of Income Difference}

The selection of method and index system for measuring residents' income difference is directly related to the accuracy and rationality of the results. Currently, there are two kinds of basic indices for studying income difference: one is absolute difference measurement, e.g. standard deviation, weighted standard deviation and average deviation; the other is relative difference measurement, e.g. extreme value deviation, variation coefficient, Gini coefficient, Lorenz curve and Theil index. Generally these indices can reflect the overall difference and changes of residents' income. The paper analyzes the income difference among Chinese rural residents in recent years through standard deviation, weighted standard deviation, average deviation, Gini coefficient and Theil index respectively. 


\subsection{Standard Deviation (S)}

Standard deviation reflects the deviation between the regional index and the corresponding arithmetic mean. The greater standard deviation, the greater absolute difference in residents' per capita income across regions. The formula is:

$$
S=\sqrt{\frac{\sum_{j}^{n}\left(y_{j}-\bar{y}\right)^{2}}{N}}
$$

Where $\mathrm{S}$ is standard deviation; $\mathrm{y}_{\mathrm{j}}$ is per capita income of rural residents in $\mathrm{j}$ region; $\bar{y}$ is average per capita income of rural residents in different regions; and $\mathrm{n}$ is the number of regions. Standard deviation is an intuitive, simple and clear index that is relatively easy to calculate. But as an arithmetic mean based on regional index deviation, it can not fully reflect the scale difference across different regions.

\subsection{Weighted Standard Deviation (Sw)}

Weighted standard deviation is also an easy and effective measurement tool for analyzing regional income difference.

$$
S_{w}=\sqrt{\sum_{j}^{n}\left(y_{j}-\bar{y}\right)^{2} * \frac{p_{j}}{p}}
$$

Where $\mathrm{y}_{\mathrm{j}}$ is the income of rural residents in $\mathrm{j}$ region; $\bar{y}$ is the average per capita income of rural resident in all regions; $P_{j}$ is the population of $j$ region; $P$ is the population of all regions; and $n$ is the number of regions. The greater $\mathrm{Sw}$ value, the greater absolute difference. Compared with standard deviation, weighted standard deviation is obviously much more resistant to the disturbance of region-division method and more stable when it comes to multi-angle analysis of the regional difference.

\subsection{Deviation (D)}

Average deviation is based on the relationship between income distribution and equivalence distribution. It is equal to the expected deviation between the overall income level and the average income level. The greater average deviation, the greater difference between income distribution and equivalence distribution, and vise versa. The formula is:

$$
D=\frac{\sum_{j}^{n}\left|y_{j}-\bar{y}\right|}{n}
$$

Where $\mathrm{D}$ is average deviation; $\mathrm{y}_{\mathrm{j}}$ is per capita income of rural residents in $\mathrm{j}$ region; $\bar{y}$ is average per capita income of rural residents in different regions; and $n$ is the number of regions. 


\subsection{Gini Coefficient (G)}

Gini coefficient is commonly used to measure inequality of income, consumption or any other things. It calculates the inequality index based on the Lorenz Curve. A practical way is to use a formula deducted by triangle method, i.e. rank income in increasing order and divide the total population into $\mathrm{n}$ income groups (no need to divide into equal parts by proportion). Assume the population of the $i$ th group account for $\mathrm{Pi}$ of total population, and the income account for Ii $(i=1,2, \ldots, n)$ of total income, then the cumulative proportion of population from 1 st to ith group $\mathrm{Mi}=\mathrm{P} 1+\ldots+\mathrm{Pi}$, and the cumulative proportion of income $\mathrm{Qi}=\mathrm{I} 1+\ldots+\mathrm{Ii}$. The formula of Gini Coefficient is:

$$
G=\sum_{i=1}^{n-1}\left(M_{i} Q_{i}-M_{i+1} Q_{i}\right)^{i}
$$

If the Gini coefficient is smaller than 0.2 , the society is quite equal in income distribution. Values between 0.2 and 0.3 indicate a high equality; between 0.3 and 0.4 , moderate inequality; between 0.4 and 0.5 , a high inequality; and greater than 0.6 , an absolute inequality.

\subsection{Theil Index (T)}

An index to measure income difference between individuals or regions. The smaller value, the less disequilibrium. A major advantage to measure inequality by the Theil index is to measure the contributions of intra-group and inter-group difference to the total difference. But note that the calculation is complicated, and the income difference represented by Theil index is largely impacted by the sample size. These shall be taken into consideration in calculating income difference with the Theil index. The total level of income difference of regions represented by the Theil index is the weighted total of the logarithm of income share against population share of each region, the weight being the income share. The formula is:

$$
T=\sum_{i=1}^{n} Y_{i} \log \frac{Y_{i}}{P_{i}}
$$

Where $\mathrm{T}$ is the Theil index, $\mathrm{n}$ is the number of regions, $\mathrm{Yi}$ is the income share of the $i$ th region, and $\mathrm{Pi}$ is the population share of the $i$ th region.

\section{Empirical Analysis}

The online version of the volume will be available in LNCS Online. Members of institutes subscribing to the Lecture Notes in Computer Science series have access to all the pdfs of all the online publications. Non-subscribers can only read as far as the 
abstracts. If they try to go beyond this point, they are automatically asked, whether they would like to order the pdf, and are given instructions as to how to do so.

\subsection{Data Source}

The data in this paper are sourced from China Statistical Yearbook 1997-2008, and basic data including population and rural residents' per capita net income are selected according to the measurement indices.

\subsection{Analysis of Absolute Difference}

According to results in Table 1, we can observe changes in absolute difference of Chinese rural residents' per capita net income, as shown in Figure 1. Figure 1 shows intuitively that the absolute difference increases year by year during 1997 and 2008 , and the change trend of the three indices are exactly the same. Standard deviation for instance, increases slowly from 910.82 of 1997 to 1252.36 of 2003 , and rapidly from 1341.89 of 2004 to 2150.52 of 2008 , showing distinct phase characteristics.

Table 1. Standard deviation, weighted standard deviation and average deviation of Chinese rural residents'per capita net income from 1997 to 2008

\begin{tabular}{|c|c|c|c|}
\hline Years & $\begin{array}{l}\text { Example Standard Deviation } \\
\text { (S) }\end{array}$ & $\begin{array}{c}\text { Weighted } \\
\text { Standard Deviation } \\
(\mathrm{Sw})\end{array}$ & $\begin{array}{c}\text { Average } \\
\text { Deviation (D) }\end{array}$ \\
\hline 1997 & 910.82 & 752.44 & 677.49 \\
\hline 1998 & 928.97 & 762.43 & 700.71 \\
\hline 1999 & 958.36 & 792.01 & 728.78 \\
\hline 2000 & 1024.03 & 859.91 & 779.98 \\
\hline 2001 & 1096.05 & 903.21 & 837.18 \\
\hline 2002 & 1179.81 & 967.12 & 897.31 \\
\hline 2003 & 1252.36 & 1045.74 & 952.59 \\
\hline 2004 & 1341.89 & 1124.25 & 1018.11 \\
\hline 2005 & 1575.31 & 1304.54 & 1178.57 \\
\hline 2006 & 1758.56 & 1451.91 & 1311.22 \\
\hline 2007 & 1940.00 & 1593.22 & 1437.33 \\
\hline 2008 & 2150.52 & 1757.13 & 1586.86 \\
\hline
\end{tabular}

Naturally, from a statistical view, the calculation of standard deviation and other indices are impacted by changes of mean value, i.e. the increase of Chinese rural residents' per capita net income can be summarized in part as the improvement of overall income per capita. Figure 2 shows that the increase of standard deviation, weighted standard deviation and average deviation of Chinese rural residents' annual per capita net income is consistent with the rising trend of overall net income per capita. It confirms that the rise of net income per capita contributes to the increase of 
absolute indices of rural residents' annual net income per capita. Accordingly, there must be certain error if standard deviation and other indices are used to study the income difference. Therefore, to eliminate the influence of this factor, relative difference indices shall be combined with absolute differences for further analysis.

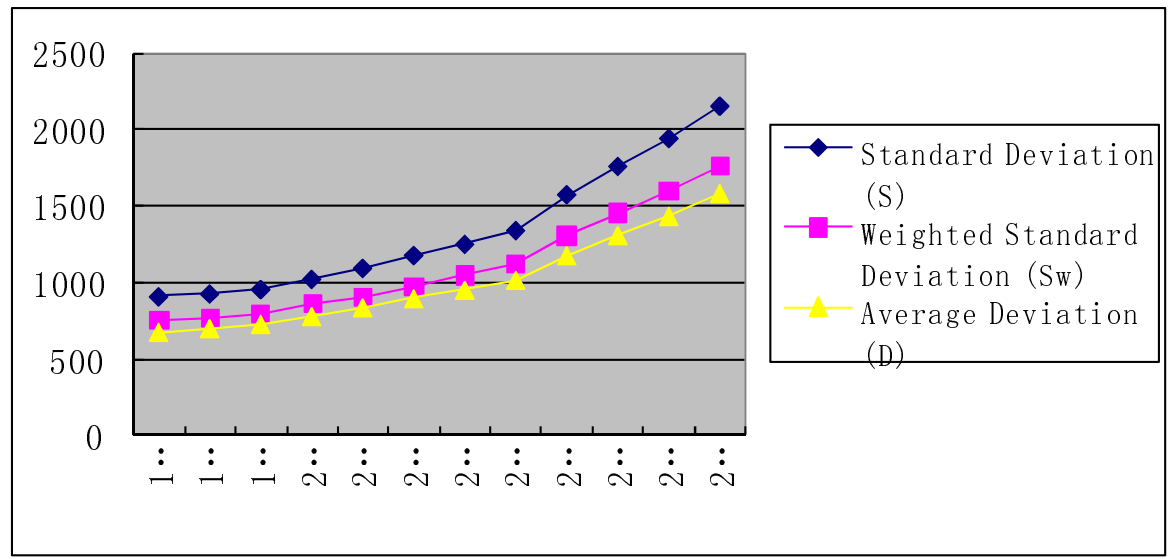

Fig. 1. Changes in absolute difference of Chinese rural residents' per capita net income from 1997 to 2008

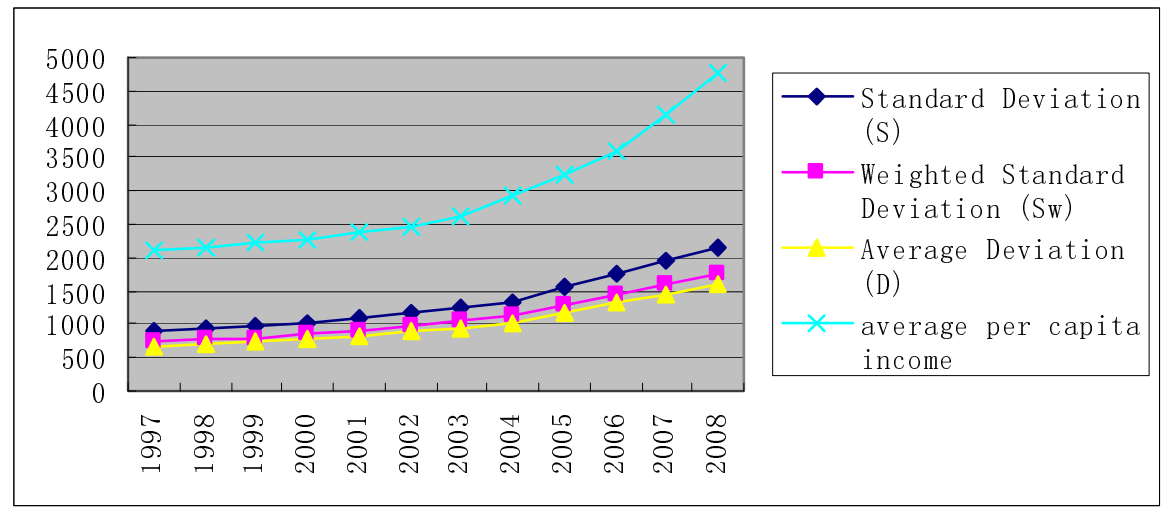

Fig. 2. Comparison of standard deviation, weighted standard deviation, average deviation with per capita net income of rural residents

\subsection{Analysis of Relative Difference}

From Table 1, Figure 3 and 4, we can see that from 1997 to 2004, the relative difference of Chinese rural residents' per capita net income evolved as follows:

First, Gini coefficient changed insignificantly from 0.401 in 1997 to 0.4097 in 2008, while obvious fluctuation appeared from 1998 to 2002 as decrease after 
increase. During the 12 years from 1997 to 2008, Gini coefficient was always around 0.41 , which shows that the income gap is relatively reasonable or a little large, and the gap tended to widen especially from 2006 to 2008.

Second, the Theil index slightly slipped from 0.3477 in 1997 to 0.3339 in 2008. It is not difficult to find out that the Theil index also fluctuates during the 12 years. In general, however, the disequilibrium of rural residents' per capita net income appears to be at average level or slightly decreasing.

Table 2. Gini coefficient and Theil index of Chinese rural residents' per capita net income from 1997 to 2008

\begin{tabular}{ccc}
\hline Years & Gini coefficient $(\mathrm{G})$ & Theil index $(\mathrm{T})$ \\
\hline 1997 & 0.4010 & 0.3477 \\
1998 & 0.4026 & 0.3511 \\
1999 & 0.4061 & 0.3513 \\
2000 & 0.4109 & 0.3441 \\
2001 & 0.4072 & 0.3564 \\
2002 & 0.3995 & 0.3629 \\
2003 & 0.3924 & 0.3668 \\
2004 & 0.3966 & 0.3538 \\
2005 & 0.3954 & 0.3564 \\
2006 & 0.4005 & 0.3582 \\
2007 & 0.4055 & 0.3449 \\
2008 & 0.4097 & 0.3339 \\
\hline
\end{tabular}

\section{Gini coefficient $(G)$}

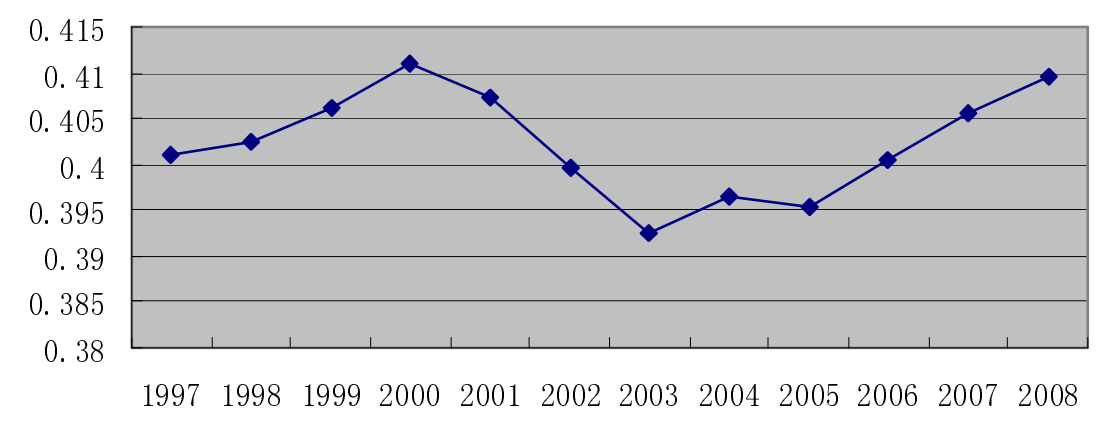

Fig. 3. Changes in Gini coefficient of Chinese rural residents' per capita net income from 1997 to 2008 


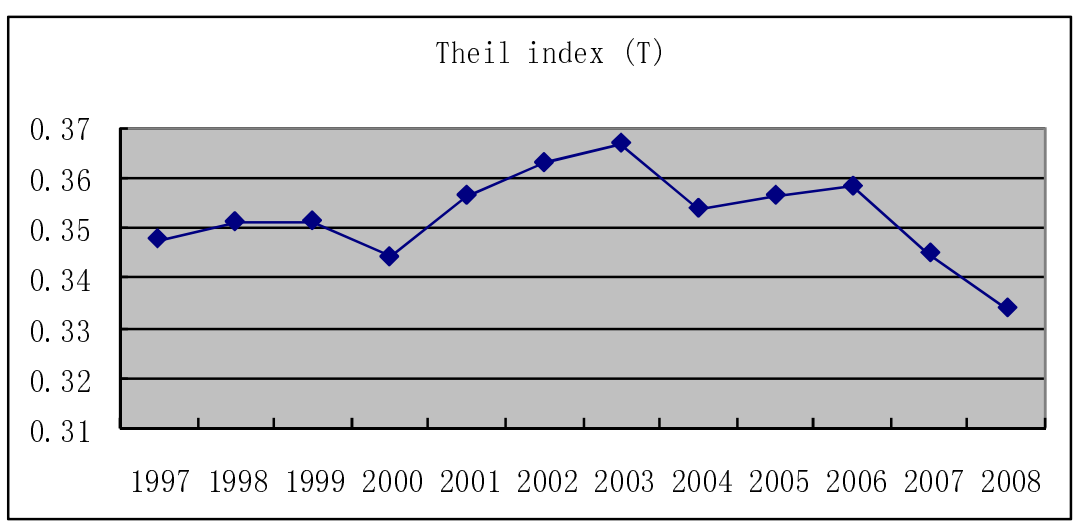

Fig. 4. Changes in Theil index of Chinese rural residents' per capita net income from 1997 to 2008

\section{Discussion and Proposal}

According to the above analysis, the absolute difference indices of Chinese rural residents' income over the 12 years from 1997 to 2008 are enlarging year by year, with obvious phase characteristics; the relative difference indices appear to be at average level in general, but the Gini coefficient tends to increase in fluctuations in recent years. Therefore, the rural residents' income gap is widening in China. Appropriate income gap can stimulate competition and break the absolute equalitarianism to promote economic development. However, as the gap is significantly widening, if we don't take corresponding measures, the trend will continue for a long term to cause excessive disparity in income, which will easily influence the social stability and hamper the economic development. Therefore, we should fully understand how rural residents' income gap may influence social economy to correctly handle the relations between efficiency and impartiality. Instead of placing our hope on the natural evolution of economy, we shall consciously and actively adjust and improve development policies for different regions to control the current rural residents' income gap in a certain range, and allow the existing gap to promote instead of hampering the economic development. To this end, we put forward the following proposals:

First, establish corresponding policies and measures to support agricultural development, increase funds to support agriculture, invest more in basic education, support rural infrastructure construction and social undertaking development, identify scientific rural industrial development strategy, and promote industrialization and urbanization in rural areas.

Second, increase rural residents' income, while narrowing their education gap and improving their quality. For low-income areas in particular, we shall encourage intellectual work and investment through macro control while actively improving farmers' income level so as to increase the income of professional technicians and managers, prevent loss of talents and resources, and ensure the development potential of low- 
income areas. Meanwhile, we shall offer preferential policies to attract the transfer of labor, technology and fund to the backward areas.

Third, regulate distribution principle, crack down illegal earnings and adjust tax system. We shall adjust and improve income distribution system, protect legitimate earnings and adopt differentiated support policies for different areas to reduce farmers' burden.

Fourth, another way to increase rural residents' income is to ensure equal opportunity to earn income. The government should ensure social members have a basic and equal start when they enter the society, i.e. each individual in the society should have equal fundamental rights including the equal right to existence, employment, education and relocation, etc.

\section{References}

1. Tao, Y.H.: Study on Regional Difference of Rural Resident Income and Its Influencing Factor. Doctoral Dissertation (2008)

2. Sun, J., Huang, H.B.: Application of Theil Index in the Analysis of Income Gap in East, Middle and West Areas. Market Modernization 500, 51 (2007)

3. Huang, T.Y., Wang, J.G.: Choice of Measurement Index System for Resident Income Gap. Contemporary Economic Research 9, 42-47 (2000)

4. Shang, Y.H.: Reason and Countermeasure Proposal for the Increase of National Gini Coefficient. Theoretical Exploration 2, 84-86 (2007) 